\title{
Compreensão de leitura e motivação para aprendizagem de alunos do ensino fundamental
}

\author{
Acácia Aparecida Angeli dos Santos¹, http://orcid.org/0000-0002-8599-7465 \\ Mayara Salgado de Moraes ${ }^{1}$, https://orcid.org/0000-0003-2308-5695 \\ Thatiana Helena Lima², https://orcid.org/0000-0001-9832-8546
}

\begin{abstract}
Resumo
O presente estudo teve como propósito identificar a relação existente entre compreensão de leitura e motivação para aprender de estudantes do ensino fundamental. Foram aplicados o Teste de Cloze e a Escala da Avaliação da Motivação para a Aprendizagem. Para tal, participaram da pesquisa 169 adolescentes do $6^{\circ}$ ao $9^{\circ}$ ano do ensino fundamental, de oito turmas diferentes, de uma única escola pública do interior do estado de São Paulo. Dentre os alunos 49,1 \% são do sexo masculino e 50,9 \% do sexo feminino, com idades entre 11 e 17 anos. Os resultados indicaram uma correlação positiva e significativa entre os escores do Teste de Cloze e os da meta aprender, e negativa significativa no que diz respeito às metas performance, aproximação e evitação. Os dados são discutidos à luz da literatura sobre o tema.
\end{abstract}

Palavras-chave: Teste de Cloze; motivação; ensino fundamental.

\section{Reading and motivation understanding for learning of fundamental teaching students}

\begin{abstract}
The present study aimed to identify the relationship between reading comprehension and motivation to learn from elementary school students. The Cloze Test and the Motivation Assessment Scale for Learning were applied. To this end, 169 adolescents from the 6th to the 9th grade of elementary school, from eight different classes, from a single public school in the interior of the state of São Paulo participated in the study. Among the students, $49.1 \%$ were male and $50.9 \%$ female, with ages ranging from 11 to 17 years. The results indicated a positive and significant correlation between the scores of the Cloze test and those of the target learning, and significant negative regarding performance, approximation and avoidance goals. The data are discussed in light of the literature on the subject.
\end{abstract}

Keywords: Cloze testing; motivation; elementary school.

\section{Comprensión de lectura y motivación para el aprendizaje de alumnos de la enseñanza primaria}

\begin{abstract}
Resumen sobre el tema.

Palabras clave: Test de Cloze; motivación; enseñanza primaria.

1 Universidade São Francisco - Itatiba - São Paulo, Brasil.

2 Universidade Federal da Bahia - Salvador - Bahia, Brasil.
\end{abstract}

En el presente estudio se tuvo como propósito identificar la relación existente entre comprensión de lectura y motivación para aprender de estudiantes de la enseñanza primaria. Se aplicó el Test de Cloze y la Escala de la Evaluación de la Motivación para el Aprendizaje. Para tal, participaron de la investigación 169 adolescentes del $6^{\circ}$ al $9^{\circ}$ año de la enseñanza primaria, de ocho grupos distintos, de una única escuela pública del interior del estado de São Paulo. Entre los alumnos el 49,1 \% son del sexo masculino y el 50,9 \% del sexo femenino, con edades entre 11 y 17 años. Los resultados indicaron una correlación positiva y significativa entre los escores del Test de Cloze y los de la meta aprender, y negativa significativa en lo que dice respeto a las metas performance, acercamiento y alojamiento. Los datos son discutidos a la luz de la literatura 


\section{Introdução}

A leitura tem um papel importante para o desenvolvimento do indivíduo como um todo, principalmente em termos sociais, visto que as habilidades de ler e escrever possibilitam o exercício de todos os papéis que possa ter na sociedade. No entanto, a leitura depende da compreensão inclui a decodificação e a interpretação de textos escritos de forma a extrair o sentido do que foi lido (Oliveira, Boruchovith, \& Santos, 2007; Joly, 2009; Witter, 2010).

A definição sobre leitura descrita nos Parâmetros Curriculares Nacionais da Língua Portuguesa - PCN (Brasil, 1997) se refere a um processo ativo de construção de significado de um texto, de acordo com seus objetivos e conhecimentos prévios do leitor sobre o assunto. A leitura exige algumas estratégias, como verificação, seleção, antecipação e inferência, que permitem ao leitor o controle do que é lido (Guidetti \& Martinelli, 2007).

A leitura e sua compreensão também permitem ao leitor tomada de decisão, entendimento da realidade como um todo, além da análise individual. É na escola que esse processo se inicia, sendo um dos principais meios de desenvolvimento da capacidade leitora. Considerando que quanto maior o treino das habilidades de leitura e compreensão, meIhor o entendimento do que está sendo lido. Assim, a ação de ler torna-se um aprendizado contínuo (Oliveira \& cols., 2007).

A compreensão de leitura é explicada como a interação entre o texto e o leitor, permitindo ao leitor fazer combinações, testar hipóteses e ter a capacidade de considerar os resultados obtidos. A leitura, portanto, não é considerada uma tarefa fácil, pois implica em uma série de coordenações e habilidades que o indivíduo deve possuir para conferir significado, organizar as palavras e buscar sentido (Silveira \& Brenelli, 2009).

Algumas características dos leitores são aspectos importantes a serem considerados na temática compreensão de leitura. Segundo Santos (2004) falhas para decodificação de informações, vocabulário restrito, falta de estratégias para aprendizagem adequadas e falta de motivação para leitura dificultam o processo de compreensão. Da mesma forma, a autora ressalta que a escola como se configura, tem papel pouco significativo na estimulação de leitura, tendo em vista que a proposta promovida se baseia apenas para o processo de ensino gramatical, distanciando práticas de leitura dos hábitos e interesses dos alunos. Ganhos em aprendizagem de leitura também se relacionam à interação dos pais no contexto escolar como agentes incentivadores.

No contexto escolar a dificuldade de compreensão de leitura tem sido identificada como um dos aspectos determinantes do fracasso escolar de alunos em vários níveis de escolarização. Pesquisas nessa área apontam para a compreensão de leitura como um dos principais problemas citados por alunos, dentre aqueles que interferem diretamente em seu desempenho acadêmico (Joly, Bonassi, Dias, Piovezan, \& Silva, 2014; Oliveira \& cols., 2007; Oliveira, Santos, \& Boruchovith, 2009; Santos, 2004).
Como forma de identificação e medição da capacidade e compreensão de leitura, um instrumento que vem sendo amplamente empregado (Santos, 2005; Cunha \& Santos, 2014; Suehiro \& Santos, 2015) é o Teste de Cloze. Ele foi criado por Taylor (1953) e tem sido considerada uma medida confiável na avaliação da compreensão de textos. Foi desenvolvida com base em princípios gestalticos, em que são enfatizados a capacidade de percepção e conhecimentos prévios do leitor.

O instrumento consiste no preenchimento de lacunas existente num texto, construído de forma estruturada. O leitor deve então preencher as lacunas por palavras que compreenda serem adequadas ao contexto (Joly \& cols., 2014; Oliveira \& cols., 2009; Suehiro \& Santos, 2015). Segundo Mota e Santos (2014), o Teste de Cloze é considerado como uma das mais eficientes medidas para avaliação da compreensão de leitura, em diversos níveis escolares. O instrumento atribui importância ao conhecimento linguístico e conceitual, além de ser preditivo ao desempenho acadêmico.

Ao lado da leitura, reconhece-se a motivação como aspectos outro aspecto que influencia o comprometimento dos alunos em tarefas de aprendizagem. A motivação é o que move o estudante a se concentrar e focar atenção suficiente, quando envolvido em tarefas de ler para aprender (Alcará \& Santos, 2013).

A relação entre a compreensão de leitura e motivação é reconhecida por alguns estudiosos, que consideram a motivação como geradora do engajamento e compromisso com a aprendizagem do aluno, o que gera resultados consistentes e duradouros (Cunha \& Boruchovitch, 2012; Santos, Alcará, \& Monteiro, 2012). Muitos estudiosos consideram como relevante a relação entre o interesse pelo aprendizado e a percepção da autonomia possível para alcançá-lo, bem como o apoio motivacional da instituição escolar para se atingir os objetivos de aprendizagem (Santos, 2004; Gomes, 2009; Cunha \& Boruchovitch, 2012; Monteiro \& Santos, 2011).

Estudos apontam (Bzuneck, 2005; Zenorini \& Santos, 2010; Zenorini, Santos, \& Monteiro, 2011) que a motivação é um dos fatores que interferem no desempenho escolar dos alunos e referem que há inúmeras variáveis, como o ambiente da sala de aula, postura e ações do professor, uso inadequado de estratégias de aprendizagem e aspectos emocionais, que podem desencadear ou inibir a motivação dos estudantes. Dentre essas variáveis, os propósitos ou as metas que a pessoa tem, são explicativas do sucesso na aprendizagem (Zenorini \& cols., 2011). No contexto escolar, Bzuneck (2009) esclarece que a motivação de estudantes se relaciona a aspectos específicos do ambiente em que está inserido, incluindo as pessoas nele presentes, o que explica a importância do professor e a forma como ele estrutura as condições de aprendizagem.

A motivação para aprendizagem envolve o investimento de recursos pessoais para o processo de aprender, sendo ele, a principal peça para um desempenho escolar bem-sucedido. Está relacionada à qualidade e não à intensidade de investimento cognitivo posto à tarefa de aprendizagem. Estudos sobre motivação no contexto escolar vêm 
crescendo recentemente e são realizados a partir de várias perspectivas teóricas, o que se entende como um construto de grande complexidade (Santos, Alcará, \& Zenorini, 2013; Scacchetti, Oliveira, \& Rufini, 2014).

A motivação para aprendizagem aqui adotada tem como base a Teoria de Metas de Realização. Bzuneck (2009) esclarece que se trata de uma continuidade da motivação para realização, desenvolvida por Lewin, Murray, McClelland e Atkinson que tem base cognitivista. Seu foco está na relação existente entre os objetivos do sujeito e o comportamento para atingir bons resultados, que indiquem sucesso, desenvolvimento de habilidades e competências, ou ainda, demonstrações de sucesso e evitação de fracasso.

A Teoria de Metas de Realização preconiza a existência de metas diversas, referindo-se especialmente ao estudante no contexto da sala de aula. Sobre a classificação dessas metas não há acordo absoluto. A literatura traz explicações sobre as características gerais de duas grandes metas e é possível verificar certa convergência semântica entre as conceituações. Zenorini e Santos (2010), apresentam nomenclaturas utilizadas por alguns autores, como meta envolvimento na tarefa e envolvimento do ego, denominadas por Duda e Nicholls (1992), ou ainda meta domínio e meta performance utilizada por Ames (1992). Para melhor entendimento, Bzuneck (2009) expõe sobre ambas, a partir da polaridade existente entre elas. São classificadas, portanto, como meta aprender e meta performance.

A meta aprender tem semelhanças com a conceituação da motivação intrínseca. Nota-se dentre as características de alunos orientados à meta aprender, envolvimento espontâneo em atividades escolares por acreditarem que o sucesso nas tarefas acarreta no desenvolvimento de suas próprias habilidades, bem como tais resultados dependam de si e da qualidade do investimento pessoal empregado para a realização. São alunos que se sentem orgulhosos ao atingirem bons resultados, porém reconhecem fracassos como desafios e aprendizagens que devem proporcionar uma melhora em suas estratégias aplicadas (Bzuneck, 2009).

Com relação à meta performance, os alunos mostram-se muito mais interessados em demonstrar o que sabem, tanto para os professores como aos próprios colegas. Santos, Alcará e Monteiro (2012) explicam que os resultados alcançados por alunos orientados à meta performance, são relacionados a expectativas que desejam apresentar publicamente, como demonstração de suas capacidades. Em oposição à meta aprender, esses alunos valorizam o reconhecimento de outras pessoas e não a aprendizagem em si. Esses alunos também apresentam baixa tolerância ao fracasso, quando mostram sentimentos de impotência, vergonha e incapacidade diante de situações que apresentaram desempenho não satisfatório.

Ainda de acordo com Santos e cols. (2012), apesar da diferença entre as duas metas, alguns estudantes podem ser orientados a ambas. Em função dessa informação, a meta performance, ainda é analisada por outros dois aspectos, sendo dividida em performance-aproximação, que é quando o aluno busca pela aparência de suas capacidades e por outro lado, performance-evitação, que diz respeito a característica de evitação de envolvimento dos alunos, que não querem se mostrar incapazes frente aos demais em situações de tarefas escolares.

Vale ressaltar que estudos sobre o desempenho acadêmico têm demonstrado que alunos mais motivados, sobretudo, os orientados ao aprendizado, são os que apresentam desempenho acadêmico mais satisfatório em geral (Zenorini \& cols., 2011). No entanto, Bzuneck (2001), ao falar sobre o esforço de estudantes para a aprendizagem, considera que nem todo aluno motivado, pode atingir resultados acadêmicos considerados satisfatórios. Tal consequência pode estar relacionada a falta de certas habilidades cognitivas das quais é dependente um bom desempenho em tarefas diversas, ou ainda o emprego de estratégias de estudo inadequadas ou falta de esforço disciplinado.

Considerando a importância da motivação para aprender, o presente estudo teve como propósito identificar a relação existente entre este construto e a compreensão de leitura. Também teve como propósito investigar possíveis diferenças, considerando as variáveis sexo e ano escolar.

\section{Método}

\section{Participantes}

Participaram 169 crianças do $6^{\circ}$ ao $9^{\circ}$ ano do ensino fundamental, de oito turmas diferentes. As turmas são de uma escola pública do interior do estado de São Paulo. Dos alunos, 49,1 \% (n=83) são do sexo masculino e 50,9 $\%(n=86)$ do sexo feminino, com idade entre 11 e 17 anos $(M=12,79 ; D P=1,201)$.

\section{Instrumentos}

Teste de Cloze "Coisas da Natureza" Santos (2005)

Trata-se de um texto preparado de acordo com a técnica de Cloze em sua versão tradicional foi utilizado e todos os $5^{\circ} \mathrm{s}$ vocábulos do texto foram omitidos. No local da omissão foi colocado um traço de tamanho proporcional à palavra excluída. Continha aproximadamente 250 vocábulos e 40 lacunas, sendo que o respondente deveria preenchê-la com a palavra que julgasse a mais adequada para dar sentido ao texto. O teste possui evidências de validade para utilização com alunos de $5^{\mathrm{a}}$ a $8^{\mathrm{a}}$ série (atuais $6^{\circ}$ ao $9^{\circ}$ ano). A prova possui evidências de validade de critério, visto que separou os alunos por etapa escolar, formando um subgrupo para cada ano, com aumento de média dos escores compatíveis com o avanço na escolarização. A pontuação é dada, considerando-se zero para erros ou espaços em branco e um ponto para cada acerto. 
Escala da Avaliação da Motivação para a Aprendizagem - EMAPRE (Zenorini \& Santos, 2010)

A escala é composta pelos fatores, meta aprender (12 itens) que se refere ao esforço e a busca pelo estudante por novos conhecimentos, meta performance-aproximação (9 itens) que identifica a preocupação do aluno em se destacar em relação aos colegas e, por fim a meta performance-evitação (7 itens), caracterizada pela preocupação do aluno em não demonstrar fragilidades diante dos colegas totalizando 28 itens. As opções de respostas apresentadas para os alunos classificam-se em $1=$ sempre, $2=$ às vezes e $3=$ nunca. $A$ pontuação máxima é de 84 e mínima de 28 pontos. Em relação aos estudos psicométricos a escala apresentou evidência de validade por meio da estrutura interna, já que a análise fatorial exploratória indicou a existência de três fatores, correspondentes às três subescalas previstas teoricamente e os itens obtiveram carga fatorial acima de 0,40. Quanto à consistência interna o alfa por dimensão foi de 0,77 para a meta aprender, 0,86 para meta performance-aproximação e 0,61 para a meta performance-evitação.

\section{Procedimento de coleta dos dados}

Primeiramente foi solicitada autorização da escola para a coleta dos dados e o projeto foi encaminhado para o comitê de ética da Universidade São Francisco. Após a aprovação do comitê de ética e os pais terem assinado o termo de consentimento livre e esclarecido, entrou-se em contato com a escola para combinar os dias e horários da aplicação dos instrumentos. Foi explicado o objetivo da pesquisa, bem como dadas as instruções de cada instrumento aos alunos. A aplicação ocorreu coletivamente, em sala de aula e com tempo aproximado de 35 minutos.

\section{Procedimento de análise dos dados}

Os dados foram inseridos em uma planilha do SPSS (versão 22) e foram utilizadas estatísticas descritivas e a inferenciais para as análises. Após a verificação da distribuição normal dos dados, foram empregados a prova de correlação de Pearson, o teste $\mathrm{t}$ de Student para verificar diferença em razão do sexo dos estudantes e a ANOVA para as comparações que envolviam a variável ano escolar.

\section{Resultados}

Inicialmente são relatadas informações descritivas sobre as médias encontradas em cada teste. Na Tabela 1 é possível observar as pontuações mínimas e máximas de cada instrumento, bem como as médias e o desvio padrão.

Tabela 1. Estatísticas descritivas de escores do Teste de Cloze e da EMAPRE.

\begin{tabular}{lccccc}
\hline & N & Mínimo & Máximo & Média & $\begin{array}{l}\text { Desvio } \\
\text { Padrão }\end{array}$ \\
\hline Cloze & 169 & 1 & 37 & 19,62 & 5,401 \\
\hline $\begin{array}{l}\text { Meta aprender } \\
\begin{array}{l}\text { Meta performance } \\
\text { aproximação }\end{array}\end{array}$ & 149 & 12 & 36 & 29,11 & 5,364 \\
$\begin{array}{l}\text { Meta performance } \\
\text { evitação }\end{array}$ & 158 & 9 & 27 & 15,69 & 4,381 \\
\hline
\end{tabular}

Tabela 2. Índice de correlação e nível de significância entre os escores do Cloze e os fatores da EMAPRE.

\begin{tabular}{ccccc}
\hline & Meta Aprender & $\begin{array}{c}\text { Meta Performance } \\
\text { Aproximação }\end{array}$ & $\begin{array}{c}\text { Meta Performance } \\
\text { Evitação }\end{array}$ \\
\hline Cloze & $r$ & 0,167 & $-0,234$ & $-0,224$ \\
& $p$ & 0,041 & 0,003 & 0,004 \\
\hline
\end{tabular}


A Tabela 1 apresenta a pontuação dos alunos para cada instrumento. Observa-se grande variabilidade entre os escores referentes à compreensão de leitura, medida pelo Teste de Cloze ( $\mathrm{DP}=5,401)$, sendo 1 (um) o número de menor acertos e 37, para alunos que mais pontuaram.

Em relação à motivação, a meta aprender também se destaca com maior pontuação entre as metas e em relação a variabilidade nas pontuações $(D P=5,364)$. Vale ressaltar que em comparação com a análise dos dados a partir da média aritmética para a EMAPRE, também calculada para este estudo, os resultados se mantiveram. A média da meta aprender $(M=2,41)$ foi prevalente em relação às demais metas (performance-aproximação $M=1,73$ e performance-evitação $M=1,61$ ).

Para responder ao objetivo principal do trabalho, verificou-se a correlação existente entre os construtos dois instrumentos, que avaliam a compreensão de leitura e a motivação para aprender. Da Tabela 2 constam os índices de correlação e os níveis de significância obtidos.

Os resultados mostraram, por um lado, a existência de correlações estatisticamente significativas e positivas, com magnitude fraca entre o Cloze e a meta aprender. Por outro lado, foi detectada correlação negativa significativa de magnitude fraca entre o Cloze e as metas performance- -aproximação e performance-evitação. Assim, quanto maior a pontuação em compreensão de leitura, menor a pontuação no fator que indica o interesse do aluno em se destacar em sala de aula (performance-aproximação) e em não querer aparentar ser incapaz frente aos colegas e professores (performance-evitação).

Para responder ao segundo objetivo da pesquisa, de comparação dos escores dos instrumentos entre os sexos dos participantes, os dados foram submetidos a análise do Teste $T$ de Student. As análises indicaram não haver diferença significativa entre os sexos, exceto em relação à meta performance-aproximação $(t=2,389 ; p=0,018)$, da escala de motivação para aprendizagem, em que a pontuação dos meninos foi significativamente maior do que a das meninas.

Em busca de verificar possíveis diferenças entre o desempenho dos alunos nos diferentes anos escolares da amostra, a análise de variância ANOVA foi realizada. A Tabela 3 apresenta os resultados.

Para a variável ano escolar, encontrou-se diferença significativa de acertos, no Teste de Cloze e para a meta Performance-Aproximação da EMAPRE. A prova post hoc de Tukey para comparação dos resultados no Teste de Cloze, dividiu a amostra em dois grupos. Os resultados constam da Tabela 4.

Tabela 3. - Análise de variância para comparação das pontuações para variável ano escolar

\begin{tabular}{lcc}
\hline & $F$ & $p$ \\
\hline Cloze & 7,816 & $<0,001$ \\
Meta Aprender & 0,114 & 0,952 \\
Meta Performance Aproximação & 4,095 & 0,008 \\
Meta Performance Evitação & 1,923 & 0,128 \\
\hline
\end{tabular}

Tabela 4. Prova de Tukey para comparação das pontuações entre ano escolar no Teste de Cloze

\begin{tabular}{lccc}
\hline & & \multicolumn{2}{c}{ Subconjunto para alfa $=0,05$} \\
\cline { 3 - 4 } Ano_escolar & $\mathrm{N}$ & 1 & 2 \\
\hline 6 & 37 & 16,14 & 19,86 \\
7 & 44 & & 20,87 \\
8 & 54 & & 21,09 \\
9 & 34 & & 0,698 \\
\hline$p$ & & 1,000 & \\
\hline
\end{tabular}


Tabela 5. Prova de Tukey para comparação das pontuações entre ano escolar para meta-performance aproximação da EMAPRE

\begin{tabular}{lccc}
\hline & & \multicolumn{2}{c}{ Subconjunto para alfa $=0,05$} \\
\cline { 3 - 4 } Ano_escolar & $\mathrm{N}$ & 1 & 2 \\
\hline 8 & 53 & 14,09 & \\
7 & 38 & 16,16 & 16,16 \\
9 & 33 & 16,24 & 16,24 \\
6 & 34 & & 17,12 \\
\hline$p$ & & & 0,759 \\
\hline
\end{tabular}

A Tabela 4 indica que os alunos do $6^{\circ}$ ano demonstraram desempenho significativamente inferior a todo o grupo estudado. Os estudantes do $9^{\circ}$ ano foram os maiores pontuadores. Em relação às demais séries apresentaram maior media, mas sem diferenças significativas.

Sobre a meta Performance Aproximação os alunos do $6^{\circ}$ ano foram os maiores pontuadores em comparação as demais turmas. A prova post hoc de Tukey dividiu a amostra em dois grupos apresentando pouca variabilidade entre os anos escolares. Os dados podem ser observados na Tabela 5.

Os resultados indicam que os estudantes do $6^{\circ}$ ano obtiveram pontuações que se diferenciaram significativamente dos alunos do $8^{\circ}$ ano, estando os alunos do $6^{\circ} \mathrm{com}$ as maiores médias. Em relação aos demais anos, alcançaram média mais elevada, mas não diferentes estatisticamente.

\section{Discussão}

O objetivo central desse trabalho foi o de investigar a relação entre a compreensão de leitura e a motivação para aprendizagem. Os resultados encontrados mostram que essa relação existe e é significativa entre os construtos. Assim os alunos que apresentam melhor desempenho no teste de compreensão de leitura tendem a ser mais motivados para aprendizagem. Da mesma forma, os alunos que menos pontuaram na compreensão de leitura, são orientados às metas performance-aproximação e performance-evitação.

Os dados sobre os alunos orientados para a meta aprender reforçam os achados de estudos de Zenorini, Santos e Monteiro (2011) sobre motivação e sua relação com o desempenho escolar de forma geral, de estudantes do ensino fundamental. Alunos com desempenho escolar satisfatório apresentam maior motivação para aprendizagem, ou seja, são estudantes que demonstram interesse e prazer pelo processo de aprendizagem ao desempenhar suas tarefas com empenho e envolvem-se com atividades desafiadoras. Importante destacar que a habilidade de compreensão de leitura tem se mostrado frequentemente atrelada ao bom rendimento acadêmico (Santos, 2004; Guidetti \& Martinelli, 2007; Oliveira \& cols., 2009; Alcará \& Santos, 2013).

Em investigação sobre as metas de realização de estudantes de $3^{\circ}$ ao $5^{\circ}$ ano do ensino fundamental, Monteiro e Santos (2011) identificaram predominância para a meta performance-evitação. Segundo as autoras, existe uma preocupação negativa dos alunos orientados a essa meta, frente aos desafios e dificuldades no ambiente acadêmico, gerando ansiedade e consequente baixo rendimento escolar. Isso sugere o exposto nesse trabalho, os alunos orientados a meta performance-evitação, apresentaram o menor desempenho no teste de Cloze de toda a amostra.

Já no que diz respeito à meta performance-aproximação, os alunos que demonstraram orientação a essa meta, apresentaram desempenho negativo no teste de avaliação da compreensão leitora. A literatura, de um modo geral, apresenta resultados contrastantes, como cita Alcará e Santos (2013), em que alunos orientados à meta performance-aproximação tendem a conquistar bons resultados, embora a motivação central dessa meta seja pela demonstração de capacidade. Tal contradição sugere o exposto por Bzuneck (2001) ao comentar que alunos motivados podem ainda apresentar desempenho escolar insatisfatório, quando lhes faltarem habilidades cognitivas necessárias para a aprendizagem.

$\mathrm{Na}$ comparação entre o sexo e o desempenho nos dois testes, as meninas apresentaram melhor desempenho na maioria dos itens avaliados, mesmo que sem diferenças significativas. Apenas para a meta performance-aproximação, a pontuação dos meninos apresentou-se significativamente maior do que das meninas, o mesmo resultado encontrado por Middleton, Kaplan, \& Midgley (2004).

Como já esperado, na avaliação de diferenças entre os anos escolares, alunos do $9^{\circ}$ ano atingiram os melhores resultados em compreensão de leitura e os alunos do $6^{\circ}$ ano apresentaram média significativamente menor em relação à toda amostra estudada. Tal resultado indica que os alunos de séries mais avançadas, por terem maior experiência 
acadêmica, naturalmente tendem a apresentar melhores resultados em compreensão de leitura, considerando que os textos aplicados em todas as turmas foram idênticos (Oliveira \& cols., 2009).

A meta aproximação foi a única dentre as metas de realização da EMAPRE na qual os estudantes se diferenciaram, na avaliação de diferenças para a variável ano escolar. Alunos do $6^{\circ}$ ano foram os que obtiveram pontuações mais elevadas nesta meta, destacando-se significativamente dos demais. Uma hipótese para tal resultado é que os alunos de séries iniciais podem estar mais motivados com as questões escolares por serem recém-ingressantes no segundo ciclo do ensino fundamental, ou seja, principiantes desta nova etapa de escolarização.

De um modo geral, os alunos mostram-se motivados para os estudos, considerando que a orientação motivacional com maior pontuação encontrada foi a meta-aprender, seguida pela performance-aproximação e por último performance-evitação. Porém, é importante mencionar o exposto por Alcará e Santos (2013) em estudo sobre a relação entre compreensão de leitura, estratégias de aprendizagem e motivação no contexto universitário. As autoras citam que tal resultado, pode estar relacionado à desejabilidade social, sendo que os alunos, ao responderem esse tipo teste, podem atribuir valores esperados a eles e não de acordo com sua realidade frente aos estudos e dificuldades.

Ao estudar habilidades envolvidas no desempenho escolar, como a compreensão de leitura, evidencia-se a necessidade de novas políticas educacionais e de incentivo, frente aos resultados insatisfatórios para os mais diversos níveis de escolarização. A habilidade para compreensão leitora em especial, é foco de muitas pesquisas relacionadas ao fracasso escolar, como fator gerador de consequências futuras em outros aspectos da vida dos indivíduos.

A realização deste trabalho trouxe importantes considerações a respeito do contexto escolar, que podem contribuir para a evolução de pesquisas de ambos os construtos e aplicações práticas no contexto de aprendizagem escolar. As correlações encontradas sugerem assim como já confirmado pela literatura, que alunos motivados para a aprendizagem são os que atingem melhor desempenho em amplo sentido na escola. Assim sendo, vale destacar a importância de novos estudos que compreendam a importância dos professores como influência positiva para o desenvolvimento de motivação intrínseca de seus alunos, por meio de estratégias motivacionais que contribuam efetivamente para a evolução em habilidade de leitura. São eles personagens significativos, quando mais preocupados em explorar a força motivacional de seus alunos, por meio de propostas mais incentivadoras do que a simples recompensa pelo alto desempenho.

Explorando as diferenças entre os sexos, salienta-se que a única significativa foi relacionada à motivação, mostrando que os meninos têm sua motivação mais orientada a fatores extrínsecos à aprendizagem. Alunos assim orientados tendem a conquistar bons resultados, embora a motivação central seja pela demonstração de capacidade.
Apesar das contribuições que este estudo apresenta, há algumas limitações a serem consideradas, especialmente no que refere ao fato de os dados terem sido coletados em uma única escola. Além disso, vale salientar que a escala de motivação empregada, foi inicialmente construída para alunos do ensino médio, para os quais se mostrou suficientemente sensível para captar o fenômeno que pretendeu avaliar. Pode-se destacar ainda que o formato tipo Likert da escala, pode ter dificultado a resposta dos estudantes por não estarem acostumados com este tipo de avaliação. Assim, é importante pensar em novas formas de aplicação, de forma a garantir que o fenômeno motivacional tenha sido captado em toda a sua dimensão.

Como continuação dessa pesquisa, sugere-se novos estudos que busquem confirmar se os meninos são motivados extrinsecamente e, se confirmado, a razão pela qual isso acontece. Também será importante a realização de estudos que adicionem novos meios de avaliação de outras variáveis que possam estar associadas a resultados positivos e negativos em compreensão de leitura, visto que é essencialmente por meio dela que se amplia o conhecimento do mundo, necessário ao cidadão crítico que a sociedade necessita.

\section{Referências}

Alcará, A.R.; Santos, A.A.A. (2013). Compreensão de leitura, estratégias de aprendizagem e motivação em universitários. Psico, 44(3), 411-420. Disponível: http://revistaseletronicas.pucrs.br/ojs/ index.php/revistapsico/article/viewArticle/12258. Recuperado: 25 abr. 2016.

Ames, C. (1992). Classrooms: Goals, structures, and student motivation. Journal of Educational Psychology, 84(3), 261-271. doi: 10.1037/0022-0663.84.3.261

Brasil, Secretaria de Educação Fundamental (1997). Parâmetros curriculares nacionais: língua portuguesa. Brasília: Secretaria de Educação Fundamental.

Bzuneck, J.A. (2001). O esforço nas aprendizagens escolares: mais do que um problema motivacional do aluno. Revista Educação e Ensino - USF, 6(1), 7- 18.

Bzuneck, J.A. (2005). A motivação dos alunos em cursos superiores. IN: Joly, M.C.R.A.; Santos, A.A.A.; Sisto, F.F. (Orgs.), Questões do cotidiano universitário (pp. 217-237). São Paulo: Casa do Psicólogo.

Bzuneck, J.A (2009). A motivação do aluno: aspectos introdutórios. IN: Boruchovtch, E.; Bzuneck, J.A. (Orgs.), A Motivação do Aluno (pp. 9-36). Rio de Janeiro: Vozes.

Cunha, N.B.; Boruchovitch, E. (2012) Estratégias de Aprendizagem e Motivação para Aprender na Formação de Professores. Revista Interamericana de Psicologia, 46(2), 247 - 254. Disponível: http://www. redalyc.org/html/284/28425280008/. Recuperado: 14 maio 2016. 
Cunha, N.B.; Santos, A.A.A. (2014). Estudo de validade do Questionário de Avaliação de Consciência Metatextual. Psicologia: Teoria e Prática, 16, 141-154. Disponível: http://pepsic.bvsalud. org/pdf/ptp/v16n1/12.pdf. Recuperado: 27 maio 2016.

Duda, J.L.; Nicholls, J.G. (1992). Dimensions of achievement motivation in schoolwork and sport. Journal of Educational Psychology, 84(3), 290 - 299. doi: 10.1037/0022-0663.84.3.290.

Gomes, M.A.M. (2009). Apoio motivacional e desenvolvimento da compreensão leitora em alunos do ensino fundamental. Educação Temática Digital, 10, 313-328. Disponível: http://ojs.fe.unicamp.br/ged/ index.php/etd/article/viewFile/2067/1872. Recuperado: 28 jun. 2016.

Guidetti, A.A.; Martinelli, S.C. (2007). Compreensão em leitura e desempenho em escrita de crianças de ensino fundamental. $P S / C$, 8(2), 175-184. Disponível: http://pepsic.bvsalud.org/pdf/psic/v8n2/ v8n2a08.pdf. Recuperado: 04 maio 2016.

Joly, M.C.R.A (2009) Estudos com o Sistema Orientado de Cloze para o Ensino Fundamental. In: Santos, A.A.A.; Boruchovitch, E.; Oliveira, K.L. (Orgs.), Cloze: um Instrumento de Diagnóstico e Intervenção (pp. 119-145). São Paulo: Casa do Psicólogo.

Joly, M.C.R.A.; Bonassi, J.; Dias, A.S.; Piovezan, N.M.; Silva, D.V. (2014). Avaliação da compreensão de leitura pelo sistema orientado de Cloze (SOC). Fractal: Revista de Psicologia, 26(1), 223-242. Disponível: http://www.scielo.br/pdf/fractal/v26n1/ v26n1a16.pdf. Recuperado: 15 jun. 2016.

Middleton, M.J.; Kaplan, A.; Midgley, C. (2004). The change in middle school students' achievement goals in mathematics over time. Social Psychology of Education, 7(3), 289-311. doi: 10.1023/B:SPOE.0000037484.86850.fa.

Monteiro, R.M.; Santos, A.A.A. (2011). Motivação para aprender: diferenças de metas de realização entre alunos do ensino fundamental. Estudos Interdisciplinares em Psicologia, 2(1), 1935. Disponível: http://pepsic.bvsalud.org/pdf/eip/v2n1/a03.pdf. Recuperado: 10 abr. 2016.

Mota, M.M.P.E.; Santos, A.A.A. (2014). O Cloze como instrumento de avaliação de leitura nas séries iniciais. Psicologia Escolar e Educacional, 18(1), 135-142. Disponível: http://www.scielo.br/pdf/ pee/v18n1/v18n1a14.pdf. Recuperado: 07 maio 2016.

Oliveira, K.L.; Boruchovitch, E.; Santos, A. A. A. (2007). Compreensão de leitura em alunos de sétima e oitava séries do ensino fundamental. Psicologia Escolar e Educacional, 11(1), 41-49. Disponível: $\quad$ http://www.scielo.br/pdf/pee/v11n1/v11n1a05.pdf. Recuperado: 20 jun. 2016.

Oliveira, K.L.; Boruchovitch, E.; Santos, A.A.A. (2009). A Técnica Cloze na Avaliação da Compreensão da Leitura. In: Santos, A.A.A.; Boruchovitch, E.; Oliveira, K.L. (Orgs.), Cloze: Um Instrumento de Diagnóstico e Intervenção (pp. 48-77). São Paulo: Casa do Psicólogo.
Santos, A.A.A. (2004). O Cloze como técnica de diagnóstico e remediação da compreensão em leitura. Interação em Psicologia, 8(2), 217-226. Disponível: http://revistas.ufpr.br/psicologia/article/ viewFile/3257/2617. Recuperado: 07 abr. de 2016.

Santos, A.A.A. (2005). O Teste de Cloze como instrumento de avaliação da compreensão em leitura. Relatório técnico. Itatiba: Universidade São Francisco.

Santos, A.A.A.; Alcará, A.R.; Monteiro, R.M (2012) A motivação para aprender na perspectiva da Teoria de Metas de Realização e Teoria da Autodeterminação. In: Boruchovitch, E.; Santos, A.A.A.; Nascimento, E. (Orgs.), Avaliação psicológica nos contextos educativo e psicossocial (pp. 149 - 179). São Paulo: Casa do Psicólogo.

Santos, A.A.A.; Alcará, A.R.; Zenorini, R.P.C. (2013). Estudos psicométricos da escala de motivação para a aprendizagem de universitários. Fractal: Revista de Psicologia, 25(3), 531-546. doi:10.1590/S1984-02922013000300008.

Scacchetti, F.A.P.; Oliveira, K.L.; Rufini, S.E. (2014). Medida de motivação para aprendizagem no Ensino Técnico Profissional. Avaliação Psicológica, 13(2), 297-305. Disponível: http:/l pepsic.bvsalud.org/scielo.php?script=sci_arttext $\&$ pid =S1677-04712014000200017. Recuperado: 15 jun. 2016.

Silveira, C.A.F.; Brenelli, R.P. (2009) A Técnica de Cloze e o jogo de regras: construindo relações e conhecimentos. In: Santos, A.A.A.; Boruchovitch, E.; Oliveira, K. L. (Orgs.), Cloze: um instrumento de diagnóstico e intervenção (pp. 249-282). São Paulo: Casa do Psicólogo.

Suehiro, A.C.B.; Santos, A.A.A. (2015). Compreensão de leitura e consciência fonológica: evidências de validade de suas medidas. Estudos de Psicologia, 32(2), 201-211. doi:10.1590/0103166X2015000200005

Taylor, W.L. (1953). Cloze procedure: a new tool for measuring readability. Journalism Quarterly, 30, 415-433. Disponível: https://www. researchgate.net/publication/232539913_Cloze_Procedure_A_ New_Tool_For_Measuring_Readability. Recuperado: 04 maio 2016.

Witter, G.P. (2010). Motivação e Leitura. In: Boruchovitch, E.; Bzuneck, J.A.; Guimarães, S.E.R. (Orgs), Motivação para aprender: Aplicações no contexto educativo (pp. 169 - 192). Petrópolis, RJ: Vozes.

Zenorini, R.P.C.; Santos, A.A.A. (2010). Escala de metas de realização como medida da motivação para aprendizagem. Revista Internacional de Psicologia, 44(2), 297-298. Disponível: http://www.redalyc.org/pdf/284/28420641010.pdf.

Zenorini, R.P.C.; Santos, A.A.A.; Monteiro, R.M.M. (2011). Motivação para aprender: relação com o desempenho de estudantes. Paidéia, 21(49), 157-164. Disponível: http://www.scielo.br/pdf/ paideia/v21n49/03.pdf. Recuperado: 29 abr. 2016. 
Recebido em: 21/11/2016

Aprovado em: 08/05/2017

\section{Sobre as autoras}

Acácia Aparecida Angeli dos Santos (acacia.angeli@gmail.com)

Doutora na Universidade de São Francisco. LAPE: Laboratório de Avaliação Psicológica e Educacional. Bragança Paulista (sede), São Paulo, São Paulo. http://orcid.org/0000-0002-8599-7465

Mayara Salgado Moraes (mayarasalgado@hotmail.com)

Graduanda e Bolsista PIBIC na Universidade de São Francisco. LAPE: Laboratório de Avaliação Psicológica e Educacional. Bragança Paulista (sede), São Paulo, São Paulo.

https://orcid.org/0000-0003-2308-5695

Thatiana Helena Lima (thatianahlima@gmail.com)

Doutora na Universidade Federal da Bahia. Salvador -BA.

https://orcid.org/0000-0001-9832-8546

(c) $\mathbf{E Y}$

License information: This is an open-access article distributed under the terms of the License (type CC-BY), which permits unrestricted use, distribution and reproduction in any medium, provided the original article is properly cited. 
